\title{
Heat and Mass Transfer Analysis of an Unsteady MHD Flow Past an Impulsively Started Vertical Plate in Presence of Thermal Radiation
}

\author{
Kamalesh Kumar Pandit, Sinam Iboton Singh, Dipak Sarma \\ Department of Mathematics, Gauhati University, Guwahati, India \\ Email address: \\ kamalesh.pandit@yahoo.co.in (K. K. Pandit), sinamisingh@gmail.com (S. I. Singh), dipaksarma11@yahoo.com (D. Sarma) \\ To cite this article: \\ Kamalesh Kumar Pandit, Sinam Iboton Singh, Dipak Sarma. Heat and Mass Transfer Analysis of an Unsteady MHD Flow Past an \\ Impulsively Started Vertical Plate in Presence of Thermal Radiation. International Journal of Fluid Mechanics \& Thermal Sciences. \\ Vol. 4, No. 2, 2018, pp. 18-26. doi: 10.11648/j.ijfmts.20180402.11
}

Received: July 28, 2018; Accepted: August 29, 2018; Published: September 30, 2018

\begin{abstract}
The present paper aims at investigating the boundary layer flow of an unsteady MHD free convection heat and mass transfer flow of a viscous, incompressible and electrically conducting fluid over an impulsively started infinite vertical plate in presence of thermal radiation. The magnetic Reynolds number is considered to be so small that the induced magnetic field can be neglected. Exact solution of the governing equations is obtained in closed form by Laplace transform technique. Expression for skin friction, Nusselt number and Sherwood number are derived. The numerical values of fluid velocity, fluid temperature and species concentration are displayed graphically whereas the numerical values of skin friction, the Nusselt number and the Sherwood number are presented in tabular form for various values of pertinent flow parameters. It is being found that the mass diffusion tends to reduce the fluid velocity whereas thermal radiation has reverse effect on the fluid velocity throughout the boundary layer region. Mass diffusion tends to reduce the species concentration whereas time has reverse effect on the species concentration throughout the boundary layer region. The radiation parameter and mass diffusion decreases the skin friction whereas Prandtl number has reverse effect on it. The radiation parameter reduces the rate of heat transfer whereas as time progress the rate of heat transfer is getting accelerated.
\end{abstract}

Keywords: MHD, Thermal Radiation, Heat and Mass Transfer, Impulsively Started Vertical Plate

\section{Introduction}

Study of MHD flow with heat and mass transfer plays an important role in biological Sciences. Effects of various parameters on human body can be studied and appropriate suggestions can be given to the persons working in hazardous areas having noticeable effects of magnetism and heat variation. Study of MHD flows also has many other important technological and geothermal applications. Some important applications are cooling of nuclear reactors, liquid metals fluid, power generation system and aero dynamics. The effects of radiation on free convection on the accelerated flow of a viscous incompressible fluid past an infinite vertical porous plate with suction has many important technological applications in the astrophysical, geophysical and engineering problem. The flow of an incompressible viscous fluid past an impulsively started infinite plate, in its own plane, was first studied by Stoke's [1]. It is also known as Rayleigh's problem in the literature.

In many industrial applications, the flow past an infinite vertical plate, started impulsively from rest, plays an important role. The flow of a viscous fluid past an infinite vertical plate moving impulsively in its own plane was first studied by Soundalgekar [2], where the effects of natural convection current due to the cooling or heating of the plate were discussed. The extension of this problem to hydromagnetic was presented by Georgantopoulos et al. [3] when the magnetic field is fixed relative to the fluid. Further, Raptis and Singh [4] studied the effect of magnetic field on this problem for another physical situation when the magnetic lines of force are fixed relative to the plate. Soundalgekar and Takhar [5] considered MHD flow and heat transfer over a semi-infinite plate under transverse magnetic field and Soundalgekar and Wavre [6] studied unsteady free 
convection flow past an infinite vertical plate with variable suction and mass transfer. Gupta et al. [7] have analyzed free convection effects on the flow past an accelerated vertical plate in an incompressible dissipative fluid. Mass transfer effects on the flow past an exponentially accelerated vertical plate with constant heat flux was studied by Jha et al. [8]. Soundalgekar and Takhar [9] have considered radiation effects on free convection flow past a semi-infinite vertical plate. Das et al. [10] have studied effects of mass transfer on flow past an impulsively started vertical infinite plate with constant heat flux and chemical reaction. Takhar et al. [11] have studied radiation effects on MHD free convection flow of a radiating gas past a semi-infinite vertical plate. Non Darcy convective boundary layer flow past a semi-infinite vertical plate in saturated porous media was studied by Takhar and Beg [12]. Radiation effect on mixed convection along a vertical plate with uniform surface temperature was studied by Hossain and Takhar [13]. Radiation and free convection flow past a moving plate was considered by Raptis and Perdikis [14]. Muthucumaraswamy et al. [15] analyzed theoretical solution of flow past an impulsively started vertical plate with variable temperature and mass diffusion. Muthucumaraswamy and Ganesan [16] have studied heat transfer effects on flow past an impulsively started semi-infinite vertical plate with uniform heat flux. Samad and Rahman [17] studied thermal radiation interaction with unsteady MHD flow past a vertical porous plate immersed in a porous medium. Some effects like radiation and mass transfer on MHD flow were studied by Muthucumaraswamy and Janakiraman [18], Chaudhary and Jain [19], Prasad et al. [20] and Muthucumaraswamy et al. [21]. Muthucumaraswamy and Vijayalakshmi [22] studied effects of heat and mass transfer on flow past an oscillating vertical plate with variable temperature. Mohamed et al. [23] have investigated combined radiation and free convection from a vertical wavy surface embedded in porous media. Unsteady MHD flow past a vertical oscillating plate with thermal radiation and variable mass diffusion was studied by Deka and Neog [24]. On the other hand Muthucumaraswamy et al. [25] studied unsteady flow past an accelerated infinite vertical plate with variable temperature and uniform mass diffusion. Radiation and mass transfer effects on MHD free convection flow past an exponentially accelerated vertical plate with variable temperature was studied by Rajesh and Verma [26]. Sangapatnam et al. [27] studied radiation and mass transfer effects on MHD free convection flow past an impulsively started isothermal vertical plate with dissipation. Suneetha et al. [28] investigated the effects of thermal radiation on the natural convective heat and mass transfer of a viscous incompressible gray absorbing-emitting fluid flowing past an impulsively started moving vertical plate with viscous dissipation. Suneetha et al. [29] studied the effects of thermal radiation on MHD free convection flow past an impulsively started vertical plate with variable surface temperature and concentration. Mahmoud [30] studied the effect of thermal radiation on unsteady MHD free convection flow past a vertical plate with temperature dependent viscosity. Seth et al. [31] studied the effects of thermal radiation and rotation on unsteady hydromagnetic free convection flow past an impulsively moving vertical plate with ramped temperature in a porous medium. Rajput and Kumar [32] studied the effect of thermal radiation on MHD flow past an impulsively started vertical plate with variable heat and mass transfer.

The radiative effects have important applications in physics and engineering processes. The radiations due to heat transfer effects on different flows are very important in space technology and high temperature processes. But very little is known about the effects of radiation on the boundary layer. Thermal radiation effects may play an important role in controlling heat transfer in polymer processing industry where the quality of the final product depends, to some extent to the heat controlling factors (Mukhopadhyay et al. [33]). High temperature plasmas, cooling of nuclear reactors, liquid metal fluids, and power generation systems are some important applications of radiative heat transfer from a vertical wall to conductive gray fluids.

The purpose of present study is to analyze the effect of thermal radiation and heat absorption parameter on an unsteady MHD free convection flow of a viscous, incompressible, electrically conducting fluid past an impulsively started vertical plate. The governing equations are first transformed into a set of normalized equations and then solved analytically by using Laplace transform technique and a general solution is obtained. The effects of different involved parameters such as magnetic field parameter, Schmidt number, Prandtl number, Grashof number for heat transfer and mass transfer and thermal radiation on the fluid velocity, temperature and concentration distributions are plotted and discussed.

\section{Mathematical Formulation}

We consider the unsteady MHD free convective heat and mass transfer flow of a viscous, incompressible, electrically conducting fluid along an impulsively started vertical plate. Coordinate system is chosen in such a way that $x^{\prime}$-axis is considered along the plate in upward direction and $y^{\prime}$-axis normal to plane of the plate in the fluid. Initially i.e., at time $t^{\prime} \leq 0$, both the fluid and plate are at rest and are maintained at a uniform temperature $T_{\infty}^{\prime}$. Also species concentration at the surface of the plate as well as at every point within the fluid is maintained at uniform concentration $C_{\infty}^{\prime}$. At time $t^{\prime}>0$, the plate is given an impulsive motion in the vertical direction with constant velocity $u_{0}$, and at the plate constant heat and mass flux are imposed. Since plate is of infinite extent in $x^{\prime}$ and $z^{\prime}$ directions and is electrically nonconducting, all physical quantities except pressure depend on $y^{\prime}$ and $t^{\prime}$ only. Also no applied or polarized voltages exist so the effect of polarization of fluid is negligible. This corresponds to the case where no energy is added or extracted from the fluid by electrical means (1973). It is assumed that the induced magnetic field generated by fluid 
motion is negligible in comparison to the applied one. This assumption is justified because magnetic Reynolds number is very small for liquid metals and partially ionized fluids which are commonly used in industrial applications [34]. In view of the above assumptions under usual Boussinesq's approximation, the unsteady flow past an infinite vertical plate is governed by the following equations:

Conservation of momentum:

$$
\frac{\partial u^{\prime}}{\partial t^{\prime}}=v \frac{\partial^{2} u^{\prime}}{\partial y^{2^{\prime}}}+g \beta\left(T^{\prime}-T_{\infty}^{\prime}\right)+g \beta^{\prime}\left(C^{\prime}-C_{\infty}^{\prime}\right)
$$

Conservation of energy:

$$
\frac{\partial T^{\prime}}{\partial t^{\prime}}=\frac{k}{\rho C p} \frac{\partial^{2} T^{\prime}}{\partial y^{2^{\prime}}}-\frac{1}{\rho C p} \frac{\partial q_{r}^{\prime}}{\partial y^{\prime}}
$$

Conservation of species concentration:

$$
\frac{\partial C^{\prime}}{\partial t^{\prime}}=D \frac{\partial^{2} C^{\prime}}{\partial y^{\prime 2}}
$$

Initial and boundary conditions for the fluid flow problem are given below:

$$
\begin{gathered}
u^{\prime}=0, T^{\prime}=T_{\infty}^{\prime}, C^{\prime}=C_{\infty}^{\prime} \text { for all } y^{\prime} \text { and } t^{\prime} \leq 0 \\
u^{\prime}=u_{0}, \frac{\partial T^{\prime}}{\partial y^{\prime}}=-\frac{q}{k}, \frac{\partial C^{\prime}}{\partial y^{\prime}}=-\frac{j^{\prime \prime}}{D} \text { at } y^{\prime}=0 \text { for } t^{\prime}>0 \\
u^{\prime} \rightarrow 0, T^{\prime} \rightarrow T_{\infty}^{\prime}, C^{\prime} \rightarrow C_{\infty}^{\prime} \text { as } y^{\prime} \rightarrow \infty \text { for } t^{\prime}>0
\end{gathered}
$$

For an optically thick fluid, in addition to emission there is also self absorption and usually the absorption co-efficient is wavelength dependent and large so we can adopt the Rosseland approximation for radiative heat flux vector $q_{r}^{\prime}$. Thus $q_{r}^{\prime}$ is given by

$$
q_{r}^{\prime}=-\frac{4 \sigma_{1}}{3 k_{1}} \frac{\partial T^{\prime 4}}{\partial y^{\prime}}
$$

We assume that the temperature differences within the flow is sufficiently small, then equation (7) can be linearized by expanding $T^{\prime 4}$ into Taylor's series about the free stream temperature $T_{\infty}^{\prime}$ and neglecting second and higher order terms in $\left(T^{\prime}-T_{\infty}^{\prime}\right)$. This results of the following approximations:

$$
T^{\prime 4} \approx 4 T_{\infty}^{\prime 3} T^{\prime}-3 T_{\infty}^{\prime 4}
$$

From (7) and (8) we have

$$
\frac{\partial q_{r}^{\prime}}{\partial y^{\prime}}=-\frac{4 \sigma_{1}}{3 k_{1}} \frac{\partial^{2} T^{\prime 4}}{\partial y^{\prime 2}}=-\frac{16 \sigma_{1} T_{\infty}^{\prime 3}}{3 k_{1}} \frac{\partial^{2} T^{\prime}}{\partial y^{\prime 2}}
$$

Thus the energy equation (3) reduces to

$$
\frac{\partial T^{\prime}}{\partial t^{\prime}}=\frac{k}{\rho C_{p}} \frac{\partial^{2} T^{\prime}}{\partial y^{\prime 2}}+\frac{16 \sigma_{1} T_{\infty}^{\prime 3}}{3 k_{1} \rho C_{p}} \frac{\partial^{2} T^{\prime}}{\partial y^{\prime 2}}
$$

In order to reduce the governing equations (1), (3) and (10), into non-dimensional form, the following dimensionless variables and parameters are introduced.

$$
\begin{gathered}
y=\frac{y^{\prime} u_{o}}{v}, u=\frac{u^{\prime}}{u_{o}}, t=\frac{t^{\prime} u_{o}^{2}}{v}, T=\frac{T^{\prime}-T_{\infty}^{\prime}}{\left(q v / K u_{o}\right)}, \\
C=\frac{C^{\prime}-C_{\infty}^{\prime}}{\left(j^{\prime \prime} v / D u_{o}\right)}, G r=\frac{g \beta v\left(q v / K u_{o}\right)}{u_{o}^{3}}, N=\frac{16 \sigma_{1} T_{\infty}^{\prime 3}}{3 K k_{1}} \\
G m=\frac{g \beta^{\prime} v\left(j^{\prime \prime} v / D u_{o}\right)}{u_{o}^{3}}, \operatorname{Pr}=\frac{\mu C p}{k}, S c=\frac{v}{D},
\end{gathered}
$$

Equation (1), (3) and (10) reduces to

$$
\begin{gathered}
\frac{\partial u}{\partial t}=\frac{\partial^{2} u}{\partial y^{2}}+G r T+G m C \\
\operatorname{Pr} \frac{\partial T}{\partial t}=(1+N) \frac{\partial^{2} T}{\partial y^{2}} \\
\frac{\partial C}{\partial t}=\frac{1}{S c} \frac{\partial^{2} C}{\partial y^{2}}
\end{gathered}
$$

The corresponding initial and boundary conditions in nondimensional form become:

$$
\begin{gathered}
u=0, T=0, C=0 \text { for all } \mathrm{y} \text { and } t \leq 0 \\
u=1, \frac{\partial T}{\partial y}=-1, \frac{\partial C}{\partial y}=-1 \text { at } y=0 \text { for } t>0 \\
u \rightarrow 0, T \rightarrow 0, C \rightarrow 0 \text { as } y \rightarrow \infty \text { for } t>0
\end{gathered}
$$

\section{Solution of the Problem}

$$
\begin{aligned}
& u(y, t)=\operatorname{erfc}\left(\frac{y}{2 \sqrt{t}}\right)+\left(A_{1}+A_{3}\right)\left\{\frac{\sqrt{t} e^{-y^{2} / 4 t}\left(4 t+y^{2}\right)}{3 \sqrt{\pi}}\right. \\
& \left.-\frac{1}{6} y\left(6 t+y^{2}\right) \operatorname{erfc}\left(\frac{y}{2 \sqrt{t}}\right)\right\} \\
& -A_{1}\left\{\frac{\sqrt{t} e^{-y^{2} \operatorname{Pr} e f f} / 4 t\left(4 t+y^{2} \operatorname{Pr} e f f\right)}{3 \sqrt{\pi}}\right. \\
& \left.-\frac{1}{6} y \sqrt{\operatorname{Pr} e f f}\left(6 t+y^{2} \operatorname{Pr} e f f\right) \operatorname{erfc}\left(\frac{y}{2} \sqrt{\frac{\operatorname{Pr} e f f}{t}}\right)\right\}
\end{aligned}
$$




$$
\begin{gathered}
-A_{3}\left\{\frac{\sqrt{t} e^{-y^{2} S c / 4 t}\left(4 t+y^{2} S c\right)}{3 \sqrt{\pi}}\right. \\
\left.-\frac{1}{6} y \sqrt{S c}\left(6 t+y^{2} S c\right) \operatorname{erfc}\left(\frac{y}{2} \sqrt{\frac{S c}{t}}\right)\right\} \\
T(y, t)=2 \sqrt{\frac{t}{\pi \operatorname{Pr} e f f}} e^{-y^{2} \operatorname{Pr} e f f} / 4 t-y \cdot \operatorname{erfc}\left(\frac{y}{2} \sqrt{\frac{\operatorname{Pr} e f f}{t}}\right) \\
C(y, t)=2 \sqrt{\frac{t}{\pi S c}} e^{-y^{2} S c / 4 t}-y \cdot \operatorname{erfc}\left(\frac{y}{2} \sqrt{\frac{S c}{t}}\right)
\end{gathered}
$$

Note that the solution given by equation (15) are for $\operatorname{Pr} e f f \neq 1$ and $S c \neq 1$. The solution for $\operatorname{Pr} e f f=1$ and $S c=1$, can be easily obtained by substituting $\operatorname{Pr}$ eff $=1$ and $S c=1$ into equations (12) and (13) and follow the same procedure as discussed above.

\subsection{Skin Friction}

The expression for the skin friction at the plate, is defined as

$$
\begin{gathered}
\tau=-\left.\frac{\partial u}{\partial y}\right|_{y=0} \\
=\frac{1}{\sqrt{\pi t}}+\left(A_{1}+A_{3}-A_{1} \sqrt{\operatorname{Pr} e f f}+A_{3} \sqrt{S c}\right) t
\end{gathered}
$$

\subsection{Nusselt Number}

The Nusselt number $\mathrm{Nu}$, which measures the rate of heat transfer at the plate is defined as

$$
\begin{gathered}
N u=-\frac{1}{T(0)}\left(\frac{\partial T^{\prime}}{\partial y^{\prime}}\right)_{y^{\prime}=0}=\frac{1}{T(0, t)} \\
=\frac{1}{2} \sqrt{\frac{\pi \cdot \operatorname{Pr} e f f}{t}}
\end{gathered}
$$

\subsection{Sherwood Number}

The Sherwood number Sh, which measures the rate of mass transfer at the plate, is given by

$$
\begin{gathered}
S h=-\frac{1}{C(0)}\left(\frac{\partial C^{\prime}}{\partial y^{\prime}}\right)_{y^{\prime}=0}=\frac{1}{C(0, t)} \\
=\frac{1}{2} \sqrt{\frac{\pi \cdot S c}{t}}
\end{gathered}
$$

\section{Results and Discussions}

In order to get the physical understand of the problem and for the purpose of analyzing the effect of thermal Grashof number (Gr), solutal Grashof number (Gm), Prandtl number $(\mathrm{Pr})$, thermal radiation parameter $(\mathrm{N})$, Schmidt number $(\mathrm{Sc})$ and time $(\mathrm{t})$ on the flow field, numerical values of the fluid velocity, fluid temperature and species concentration in the boundary layer region were computed and are displayed graphically versus boundary layer co-ordinate y in Figures 111. The numerical values of skin friction, heat transfer coefficient in terms of Nusselt number $(\mathrm{Nu})$ and mass transfer co-efficient in terms of Sherwood number (Sh) are depicted in Tables 1-6. During the course of numerical calculations of the fluid velocity, the temperature and the species concentration, the values of the Prandtl number are chosen for air at $25^{\circ} \mathrm{C}$ and one atmospheric pressure $(\operatorname{Pr}=0.71)$, Mercury $(\operatorname{Pr}=0.025)$, electrolytic solution $(\mathrm{Pr}=1.0)$ and water $(\mathrm{Pr}=7.0)$. To focus our attention on numerical values of the results obtained in the study, the values of Sc are chosen for the gases representing diffusing chemical species of most common interest in air, namely, hydrogen $(\mathrm{Sc}=0.22)$, water-vapour $(\mathrm{Sc}=0.60)$ and ammonia $(\mathrm{Sc}=0.78)$. To examine the effect of parameters related to the problem on the velocity field, the skin friction numerical computation are carried out at $\mathrm{Pr}=0.71$ and $\mathrm{Sc}=0.22$.

Figures 1 and 2 depict the influence of thermal and concentration buoyancy forces on fluid velocity. It is perceived from Figures 1 and 2 that the fluid velocity increases on increasing values of $\mathrm{Gr}$ and $\mathrm{Gm}$ throughout the boundary layer region. Gr represents the relative strength of thermal buoyancy force to viscous force and Gm represents the relative strength of concentration buoyancy force to viscous force. Therefore, $\mathrm{Gr}$ increases on increasing the strengths of thermal buoyancy force whereas $\mathrm{Gm}$ increases on increasing the strength of concentration buoyancy force. In this problem, natural convection flow induced due to thermal and concentration buoyancy forces; therefore, thermal and concentration buoyancy force tends to accelerate the fluid velocity throughout the boundary layer region which is clearly evident from Figures 1 and 2.

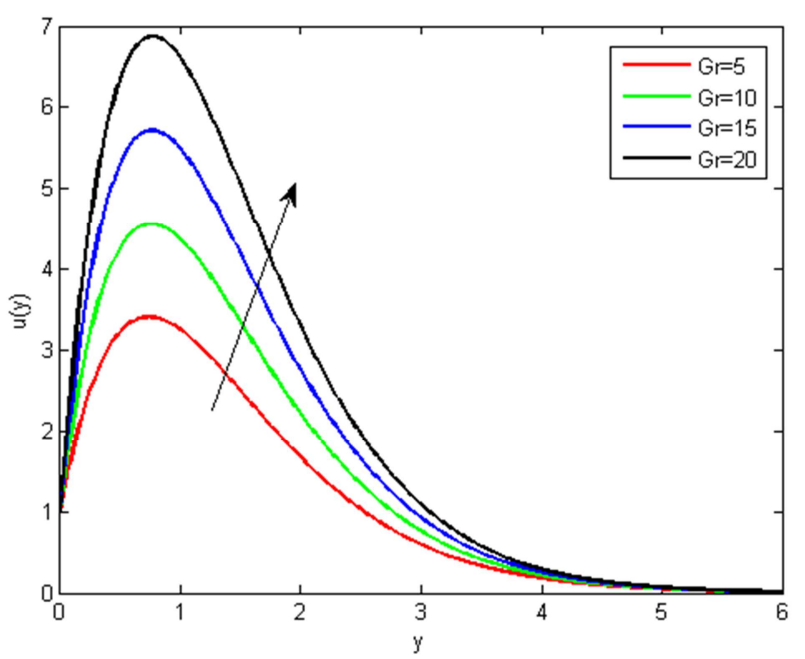

Figure 1. Fluid velocity u against y for different values of Gr. 


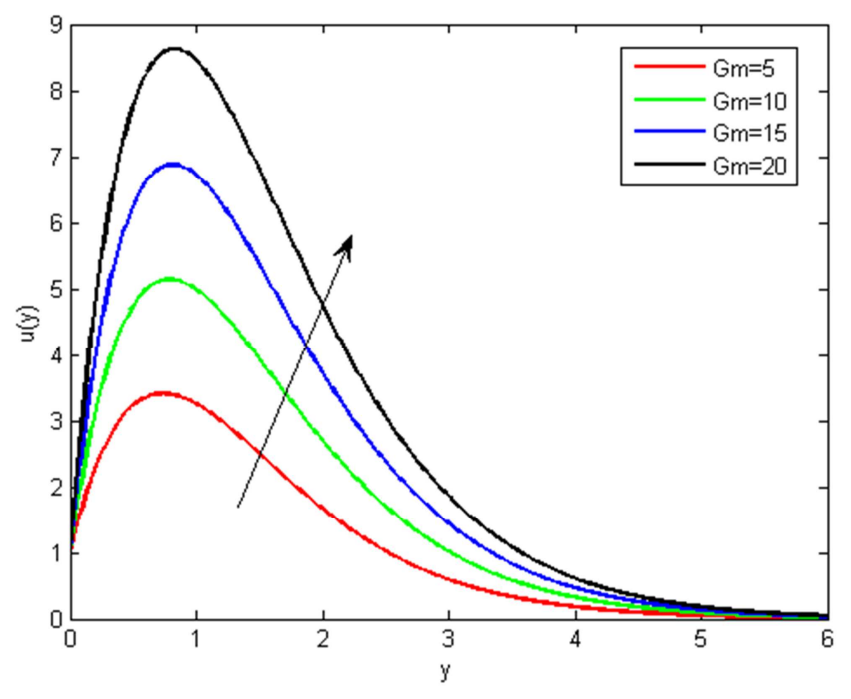

Figure 2. Fluid velocity u against y for different values of $\mathrm{Gm}$.

The influence of Schmidt number (Sc) on the fluid velocity and concentration profiles are depicted in Figures 3 and 4 respectively. It is noticed from Figures 3 and 4 that, fluid velocity and concentration profiles decreases on increasing the values of Sc. The Schmidt number embodies the ratio of the momentum to the mass diffusivity. The Schmidt number therefore quantifies the relative effectiveness of momentum to mass transport by diffusion in the hydrodynamic (velocity) and concentration (species) boundary layers. As the Schmidt number increases the concentration decreases. This cause the concentration buoyancy effects to decrease yielding a reduction in the fluid velocity. The reductions in the velocity and concentration profiles are accompanied by simultaneous reductions in the velocity and concentration boundary layers. These behaviours are clear from Figures 3 and 4.

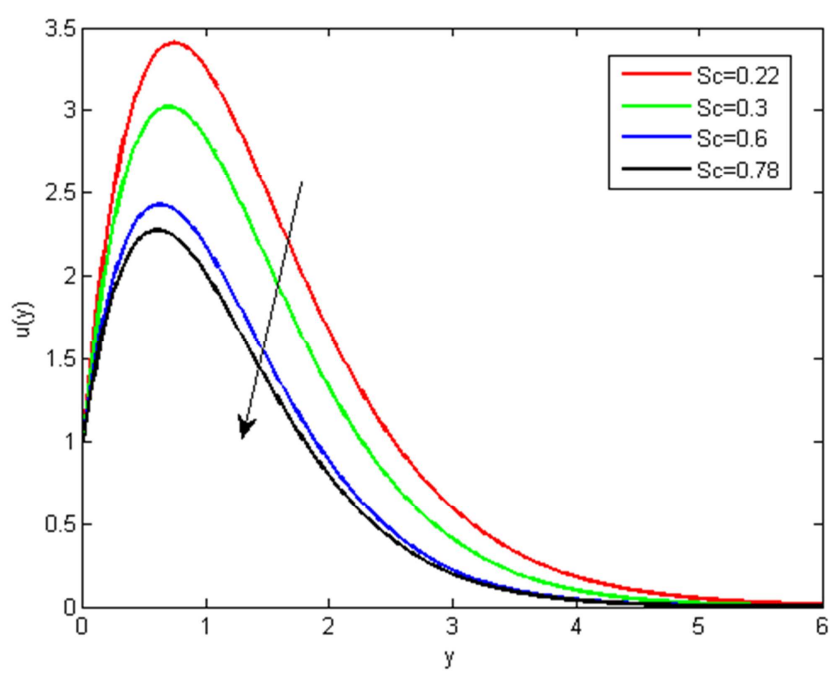

Figure 3. Fluid velocity u against y for different values of Sc.

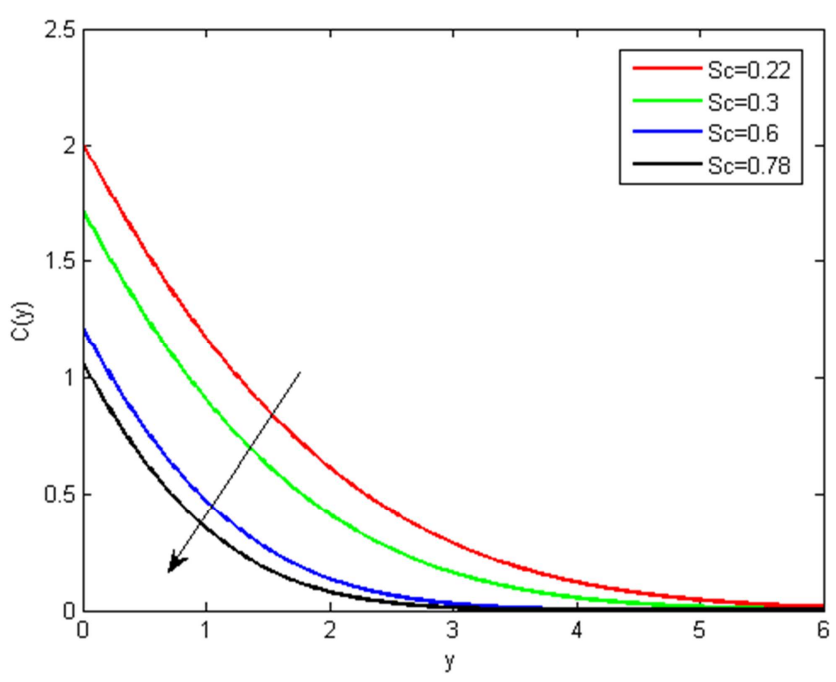

Figure 4. Species concentration C against y for different values of Sc.

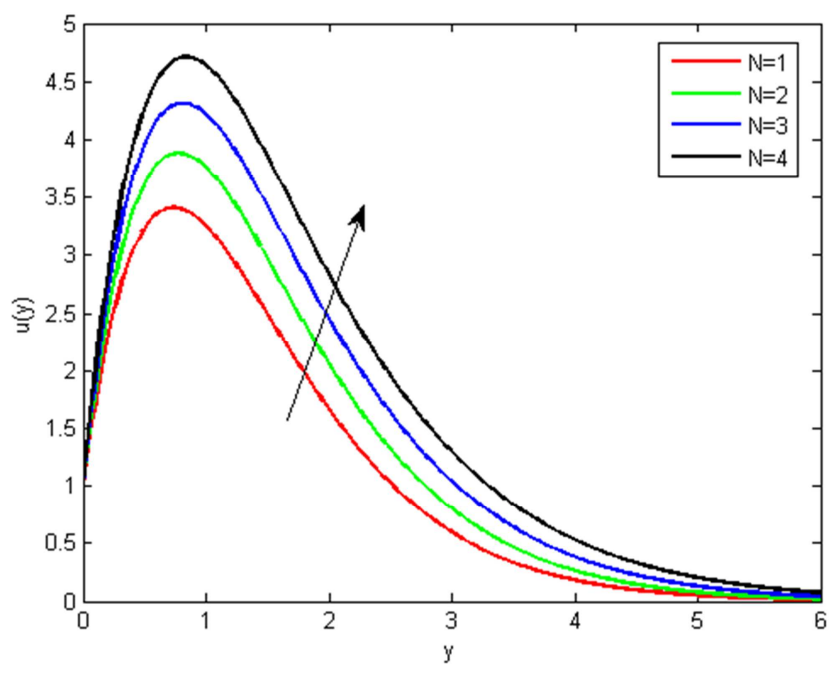

Figure 5. Fluid velocity u against $y$ for different values of $N$.

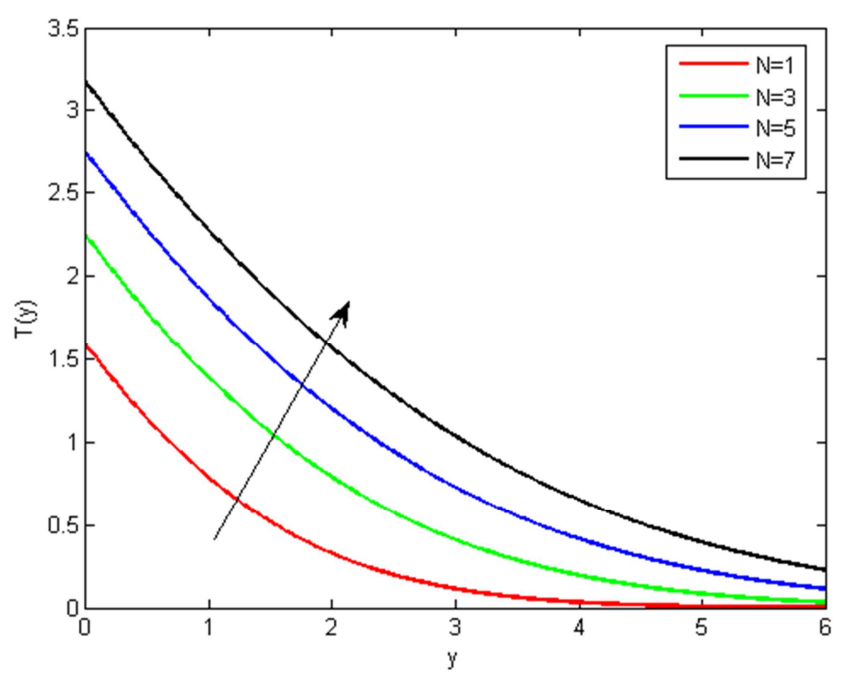

Figure 6. Fluid temperature T against y for different values of $N$.

Figures 5 and 6 demonstrate the influence of thermal radiation parameter $(\mathrm{N})$ on fluid velocity and fluid temperature respectively. It is evident from Figures 5 and 6 
that, the thermal radiation has accelerating effect on fluid velocity and fluid temperature. The rate of radiative heat transfer to the fluid decreases and consequently, the fluid velocity increases with increasing values of thermal radiation parameter for other fixed parameters.

The influence of Prandtl number (Pr) on the fluid velocity and fluid temperature are depicted in Figures 7 and 8 respectively. It is evident from Figure 7 and 8 that, fluid velocity $u(y, t)$ and fluid temperature $\mathrm{T}$ decreases on increasing Pr. An increase in Prandtl number reduces the thermal boundary layer thickness. Prandtl number signifies the ratio of momentum diffusivity to thermal diffusivity. It can be noticed that as Pr decreases, the thickness of the thermal boundary layer becomes greater than the thickness of the velocity boundary layer according to the well-known relation $\delta T / \delta \cong 1 / \operatorname{Pr}$ where $\delta T$ the thickness of the thermal boundary layer and $\delta$ the thickness of the velocity boundary layer, so the thickness of the thermal boundary layer increases as Prandtl number decreases and hence temperature profile decreases with increase in Prandtl number. In heat transfer problems, the Prandtl number controls the relative thickening of momentum and thermal boundary layers. When Prandtl number is small, it means that heat diffuses quickly compared to the velocity (momentum), which means that for liquid metals, the thickness of the thermal boundary layer is much bigger than the momentum boundary layer. Hence Prandtl number can be used to increase the rate of cooling in conducting flows.

Figures 9, 10 and 11 illustrate the influence of time on fluid velocity, fluid temperature and species concentration respectively. It is evident from Figures 9, 10 and 11 that, the fluid velocity, fluid temperature and species concentration are getting accelerated with the progress of time throughout the boundary layer region. Also it may be noted that, unabated mass diffusion into the fluid stream, the molar concentration of the mixture rises with increasing time and so there is an enhancement in species concentration with the progress of time throughout the boundary layer region.

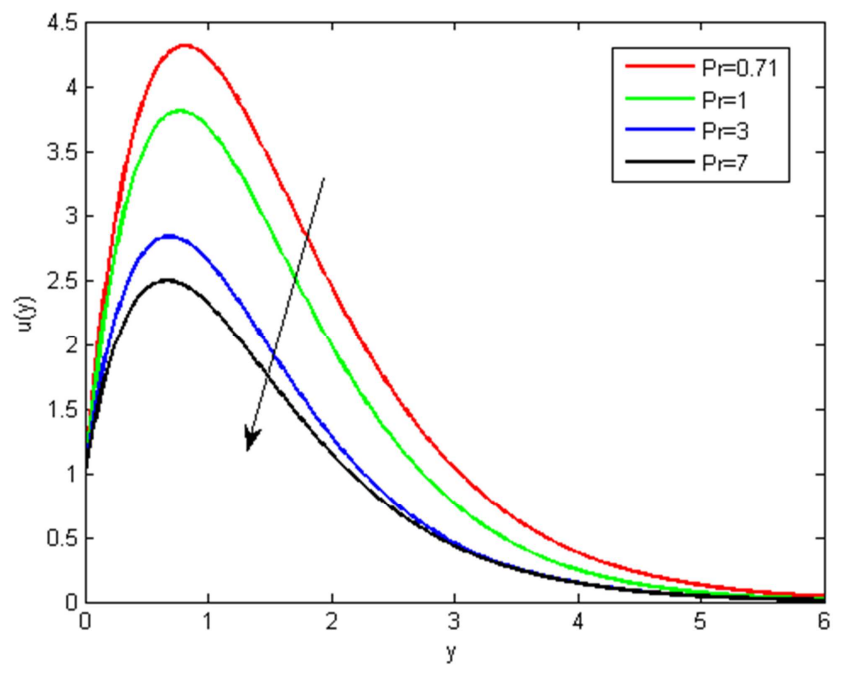

Figure 7. Fluid velocity u against y for different values of Pr.

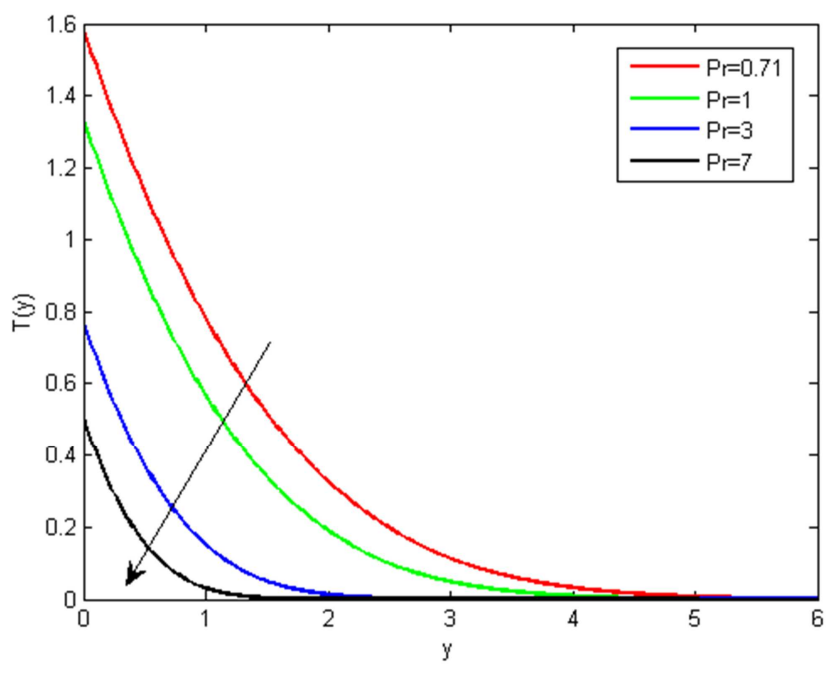

Figure 8. Fluid temperature T against y for different values of Pr.

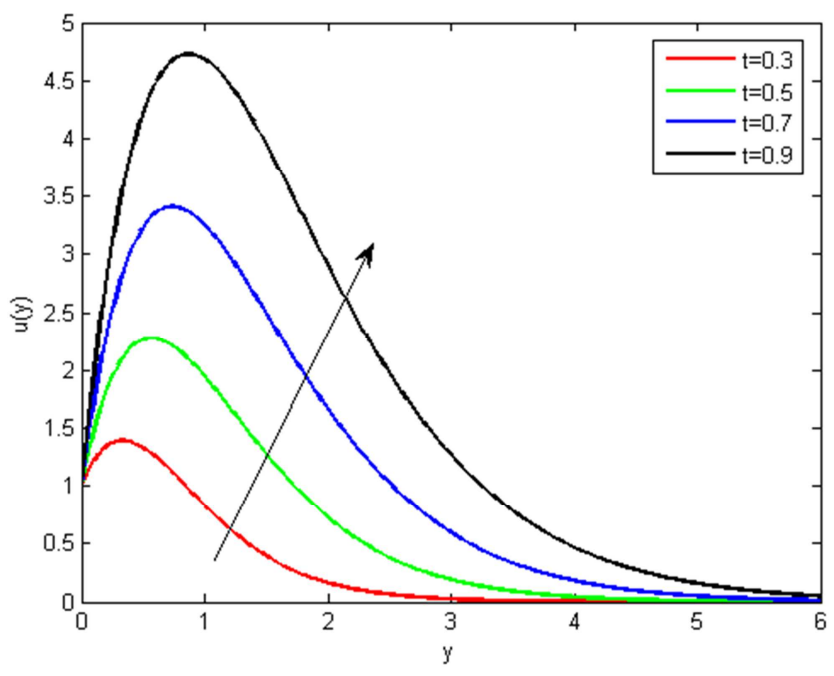

Figure 9. Fluid velocity u against y for different time $t$.

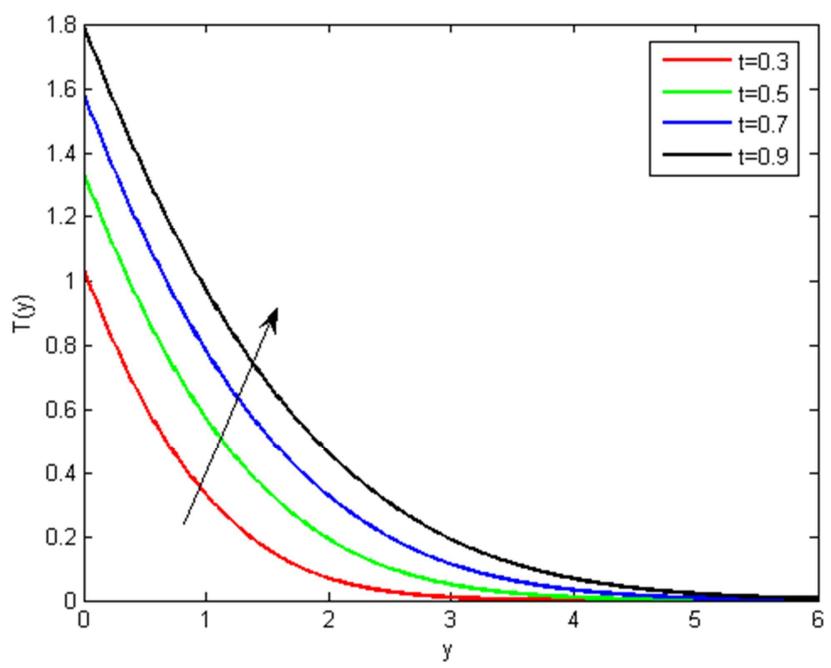

Figure 10. Fluid temperature T against y for different time $t$. 


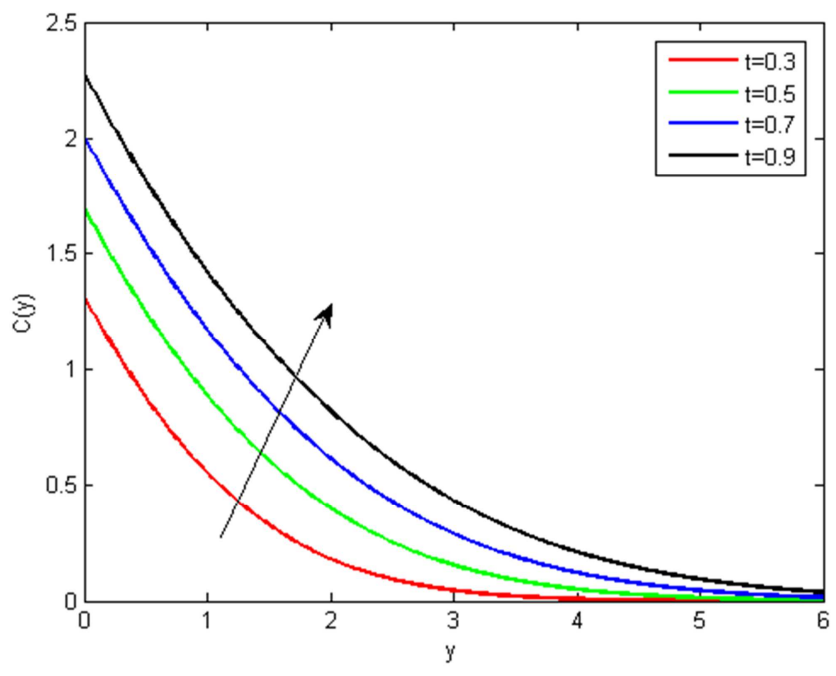

Figure 11. Species concentration $C$ against $y$ for different time $t$.

Table 1. Skin Friction when $\operatorname{Pr}=0.71, S c=0.22, N=1, t=0.5$.

\begin{tabular}{lllll}
\hline & $\mathbf{G m}$ & \multicolumn{3}{l}{$\tau$} \\
\cline { 2 - 5 } $\mathbf{G r}$ & 5 & 10 & 15 & 20 \\
\hline 5 & -5.4181 & -10.8177 & -16.8148 & -23.5584 \\
10 & -8.1800 & -14.7721 & -21.2975 & -28.3560 \\
15 & -10.9419 & -18.7265 & -25.7802 & -33.1537 \\
20 & -13.7038 & -22.6809 & -30.2629 & -37.9513 \\
\hline
\end{tabular}

Table 2. Skin Friction when $G r=5, G m=5, P r=0.71, S c=0.22$.

\begin{tabular}{lllll}
\hline & $\mathbf{t}$ & $\boldsymbol{\tau}$ & & \\
$\mathbf{N}$ & $\mathbf{0 . 5}$ & $\mathbf{1}$ & $\mathbf{1 . 5}$ & $\mathbf{2}$ \\
\hline 1 & -5.4181 & -6.9871 & -8.0615 & -8.8894 \\
3 & -11.8677 & -15.0058 & -17.1545 & -18.8104 \\
5 & -18.1872 & -22.8943 & -26.1174 & -28.6012 \\
7 & -24.4649 & -30.7411 & -35.0385 & -38.3502 \\
\hline
\end{tabular}

Table 3. Skin Friction when $G r=5, G m=5, N=1, t=0.5$.

\begin{tabular}{|c|c|c|c|c|}
\hline & $\tau$ & & & \\
\hline Pr & 0.22 & 0.3 & 0.6 & 0.78 \\
\hline 0.71 & -5.4181 & -6.1585 & -7.9580 & -10.9958 \\
\hline 1 & -4.7798 & -5.4776 & -6.3100 & -9.2933 \\
\hline 7 & -3.1626 & -4.4143 & -5.7968 & -6.5309 \\
\hline
\end{tabular}

The numerical values of the skin friction $\tau$, computed from the analytical expression (20), is presented in tabular form for various values of $\mathrm{Gr}, \mathrm{Gm}, \mathrm{Pr}, \mathrm{Sc}, \mathrm{N}$ and $\mathrm{t}$ in Tables 1-3. It is evident from Tables 1-3 that, the skin friction increases on increasing $\operatorname{Pr}$ whereas it decreases on increasing Gr, Gm, N, Sc and t. This implies that, thermal diffusion has the tendency to enhance the skin friction coefficient whereas thermal buoyancy force, concentration buoyancy force, thermal radiation, mass diffusion and time has the tendency to reduce the skin friction coefficient at the plate.

The numerical values of heat transfer coefficient in terms of Nusselt number $(\mathrm{Nu})$, computed from the analytical expression (21), and are presented in tabular form for various values of $\mathrm{Pr}, \mathrm{N}$ and $\mathrm{t}$ in Tables 4-5. It is noticed from Tables 4 and 5 that, Nusselt number increases on increasing time whereas it decrease on increasing Prandtl number and thermal radiation. This implies that, time tends to enhance rate of heat transfer at the plate whereas Prandtl number and thermal radiation has the tendency to reduce the rate of heat transfer at the plate.

The numerical values of mass transfer coefficient in terms of Sherwood number (Sh), computed from the analytical expression (22), and are presented in tabular form for various values of Sc and $t$ in Tables 6. It is revealed from Table 6 that, the rate of mass transfer increases on increasing $t$ whereas it decreases on increasing Schmidt number. This implies that, time tends to enhance rate of mass transfer at the plate whereas mass diffusion has the tendency to reduce the rate of mass transfer at the plate.

Table 4. Nusselt Number when $N=1$.

\begin{tabular}{|c|c|c|c|c|}
\hline \multirow{2}{*}{${ }_{P r}^{t}$} & \multicolumn{4}{|l|}{$\mathbf{N u}$} \\
\hline & 0.5 & 1 & 1.5 & 2 \\
\hline 0.71 & 0.7467 & 0.8862 & 1.5350 & 2.3447 \\
\hline 1 & 0.5280 & 0.6267 & 1.0854 & 1.6580 \\
\hline 3 & 0.4311 & 0.5117 & 0.8862 & 1.3537 \\
\hline 7 & 0.3734 & 0.4431 & 0.7675 & 1.1724 \\
\hline
\end{tabular}

Table 5. Nusselt Number when $\operatorname{Pr}=0.71$.

\begin{tabular}{lllll}
\hline $\mathbf{N}$ & $\mathbf{t}$ & $\mathbf{N u}$ & & \\
\cline { 2 - 5 } & $\mathbf{0 . 5}$ & $\mathbf{1}$ & $\mathbf{1 . 5}$ & $\mathbf{2}$ \\
\hline 1 & 0.7467 & 0.5280 & 0.4311 & 0.3734 \\
3 & 0.5280 & 0.3734 & 0.3049 & 0.2640 \\
5 & 0.4311 & 0.3049 & 0.2489 & 0.2156 \\
7 & 0.3734 & 0.2640 & 0.2156 & 0.1867 \\
\hline
\end{tabular}

Table 6. Sherwood Number for different values of Sc.

\begin{tabular}{lllll}
\hline \multirow{2}{*}{$\mathbf{S c}$} & $\mathbf{t}$ & $\mathbf{S h}$ & & \\
\cline { 2 - 5 } & $\mathbf{0 . 5}$ & $\mathbf{1}$ & $\mathbf{1 . 5}$ & $\mathbf{2}$ \\
\hline 0.22 & 0.5879 & 0.6865 & 0.9708 & 1.1069 \\
0.3 & 0.4157 & 0.4854 & 0.6865 & 0.7827 \\
0.6 & 0.3394 & 0.3963 & 0.5605 & 0.6391 \\
0.78 & 0.2939 & 0.3432 & 0.4854 & 0.5534 \\
\hline
\end{tabular}

\section{Conclusion}

The unsteady MHD natural convection flow with heat and mass transfer of a viscous, incompressible, electrically conducting and thermal radiating fluid over an impulsively started vertical plate.. Exact solutions of the governing equations were obtained using Laplace transform technique. A comprehensive set of graphical for the fluid velocity, fluid temperature and species concentration is presented and their dependence on some physical parameters is discussed. Significant finding are as follows:

1. Mass diffusion tends to reduce the fluid velocity whereas thermal radiation has reverse effect on the fluid velocity throughout the boundary layer region.

2. The fluid velocity decreases with the increase of Prandtl number throughout the boundary layer region.

3. Thermal radiation and time has the tendency to enhance the fluid temperature whereas Prandtl number has the reverse effect on the fluid temperature throughout the boundary layer region.

4. Mass diffusion tends to reduce the species concentration whereas time has reverse effect on the species concentration throughout the boundary layer region. 
5. Thermal radiation and mass diffusion tends to reduce the skin friction whereas Prandtl number has reverse effect on it.

6. Thermal radiation and Prandtl number tends to reduce the rate of heat transfer whereas as time progress the rate of heat transfer is getting accelerated.

7. Mass diffusion tends to reduce the rate of mass transfer whereas as time progress the rate of mass transfer is getting accelerated.

\section{Acknowledgements}

The authors would like to thank the Gauhati University, Dept. of Mathematics for their support in the development of the paper.

\section{Nomenclature}

$\mathrm{C}^{\prime} \quad$ species concentration

$\mathrm{Cp} \quad$ specific heat at constant pressure

D the coefficient of mass diffusivity

g acceleration due to gravity

Gm the solutal Grashof number

Gr the thermal Grashof number

$j^{\prime \prime} \quad$ mass flux per unit area at the plate

$\mathrm{k} \quad$ thermal conductivity

$k_{1} \quad$ Rosseland mean absorption co-efficient

$\mathrm{N}$ thermal radiation parameter

Pr Prandtl number

$q \quad$ heat flux per unit area at the plate

$\mathrm{q}_{r}^{\prime} \quad$ radiative heat flux vector

Sc Schmidt number

$\mathrm{T}^{\prime} \quad$ the temperature of the fluid

$u^{\prime} \quad$ the fluid velocity in the $x^{\prime}$-direction

$u_{0} \quad$ velocity of the plate

$\beta \quad$ the volumetric coefficient of thermal expansion

$\beta^{\prime} \quad$ the volumetric coefficient of expansion for

concentration

$\rho \quad$ the fluid density

$\sigma \quad$ electrical conductivity

$\sigma_{1} \quad$ Stefan-Boltzmann constant

$v \quad$ the kinematic viscosity

\section{References}

[1] Stokes G. G. (1851). On the effect of internal friction of fluids on the motion of pendulums. Camb. Phil. Trans. IX, 8-106.

[2] Soundalgekar V. M. (1977). Free convection effects on the Stokes problem for an infinite vertical plate. ASME J. Heat Transfer, 99, 499-501.

[3] Georgantopoulos G. A., Douskos C. N., Kafousias N. G. and Goudas C. L. (1978). Lett. in Heat and Mass Transfer, 6, 379.

[4] Raptis A. and Singh A. K. (1983). MHD free convection flow past an accelerated vertical plate. Int. Comm. Heat Mass Transfer, 10, 313.
[5] Soundalgekar V. M. and Takhar H. S. (1977). On MHD flow and heat transfer over a semi-infinite plate under transverse magnetic field. Nuclear Engineering and Design, 42, 233-236.

[6] Soundalgekar V. M. and Wavre P. D. (1977). Unsteady free convection flow past an infinite vertical plate with variable suction and mass transfer. Int. J. Heat Mass Transfer, 20, 1375-1380.

[7] Gupta A. S., Pop I. and Soundalgekar V. M. (1979). Free convection effects on the flow past an accelerated vertical plate in an incompressible dissipative fluid. Rev. Roum. Sci. Techn.-Mec. Apl., 24, 561-568.

[8] Jha B. K., Prasad R. and Rai S. (1991). Mass transfer effects on the flow past an exponentially accelerated vertical plate with constant heat flux. Astrophysics and Space Science, 181, $125-134$.

[9] Soundalgekar V. M. and Takhar H. S. (1993). Radiation effects on free convection flow past a semi-infinite vertical plate. Modeling, Measurement and Control B, 51, 31-40.

[10] Das U. N., Deka R. K. and Soundalgekar V. M. (1994). Effects of mass transfer on flow past an impulsively started vertical infinite vertical plate with constant heat flux and chemical reaction. Forschung in Ingenieurwesen, 60, 284-287.

[11] Takhar H. S., Gorla R. S. and Soundalgekar V. M. (1996). Radiation effects on MHD free convection flow of a radiating gas past a semi-infinite vertical plate. Int. J. Numerical Methods Heat Fluid Flow, 6, 77-83.

[12] Takhar H. S. and Beg O. A. (1996). Non-Darcy convective boundary layer flow past a semi-infinite vertical plate in saturated porous media. Heat Mass Transfer, 32, 33-44.

[13] Hossain M. A. and Takhar H. S. (1996). Radiation effect on mixed convection along a vertical plate with uniform surface temperature. Heat and Mass Transfer, 31, 243-248.

[14] Raptis A. and Perdikis C. (1999). Radiation and free convection flow past a moving plate. Int. J. of App. Mech. and Eng., 4, 817-821.

[15] Muthucumaraswamy R., Ganesan P. and Soundalgekar V. M. (2000). Theoretical solution of flow past an impulsively started vertical plate with variable temperature and mass diffusion. Forschung im Ingenieurwesen, 66, 147-151.

[16] Muthucumaraswamy R. and Ganesan P. (2002). Heat transfer effects on flow past an impulsively started semi-infinite vertical plate with uniform heat flux. Nuclear Engineering and Design, 215, 243-250.

[17] Samad Md. A. and Rahman M. M. (2006). Thermal radiation interaction with unsteady MHD flow past a vertical porous plate immersed in a porous medium. Journal of Naval Architecture and Marine Engineering, 3, 7-14.

[18] Muthucumaraswamy R. and Janakiraman (2006). MHD and Radiation effects on moving isothermal vertical plate with variable mass diffusion. Theor. Appl. Mech., 33 (1), 17-29.

[19] Chaudhary and Jain A. (2007). Combined heat and mass transfer effects on MHD free convection flow past an oscillating plate embedded in porous medium. Rom. Journal Phys., 52 (5-7), 505-524.

[20] Prasad V. R., Reddy N. B. and Muthucumaraswamy R. (2007). Radiation and mass transfer effects on twodimensional flow past an impulsively started infinite vertical plate. Int. J. Thermal Sci., 46 (12), 1251-1258. 
[21] Muthucumaraswamy R., Sathappan K. E. and Natarajan R. (2008). Mass transfer effects on exponentially accelerated isothermal vertical plate. Int. J. of Appl. Math. and Mech., 4 (6), 19-25.

[22] Muthucumaraswamy R. and Vijayalakshmi A. (2008). Effects of heat and mass transfer on flow past an oscillating vertical plate with variable temperature. Int. J. of Appl. Math and Mech., 4 (1), 59-65.

[23] Mohamed R. A., Mahdy A. and Hady F. M. (2008). Combined radiation and free convection from a vertical wavy surface embedded in porous media. Int. J. of Appl. Math and Mech., 4 (1), 49-58.

[24] Deka R. K. and Neog B. C. (2009). Unsteady MHD flow past a vertical oscillating plate with thermal radiation and variable mass diffusion. Chamchuri Journal of Mathematics, 1 (2), 7992.

[25] Muthucumaraswamy R., Raj M. S. and Subramanian V. S. A. (2009). Unsteady flow past an accelerated infinite vertical plate with variable temperature and uniform mass diffusion. Int. J. of Appl. Math and Mech., 5 (6), 51-56.

[26] Rajesh V. and Verma V. K. S. (2009). Radiation and mass transfer effects on MHD free convection flow past an exponentially accelerated vertical plate with variable temperature. ARPN J. of Engg. and Appl. Sci., 4 (6), 20-26.

[27] Sangapatnam S., Nandanoor B. R. and Vallampati R. P. (2009). Radiation and mass transfer effects on MHD free convection flow past an impulsively started isothermal vertical plate with dissipation. Thermal Science, 13 (2), 171-181.

[28] Suneetha S., Bhaskar Reddy N. and Ramachandra Prasad V.
(2009). Effects of thermal radiation on the natural convective heat and mass transfer of a viscous incompressible gray absorbing-emitting fluid flowing past an impulsively started moving vertical plate with viscous dissipation. Thermal Science, 13 (2), 71 - 181.

[29] Suneetha S., Bhaskar Reddy N., Ramachandra Prasad V. (2008). Thermal radiation effects on MHD free convection flow past an impulsively started vertical plate with variable surface temperature and concentration. J. Naval Archit. Marine Eng., 5 (2), 57-70.

[30] Mahmoud M. A. A. (2009). Thermal radiation effect on unsteady MHD free convection flow past a vertical plate with temperature dependent viscosity. Can J. Chem. Eng., 87, 4752.

[31] Seth G. S., Nandkeolyar R. and Ansari Mds. (2013). Effects of thermal radiation and rotation on unsteady hydromagnetic free convection flow past an impulsively moving vertical plate with ramped temperature in a porous medium. J. Appl. Fluid Mech., 6, 27-38.

[32] Rajput U. S. and Kumar S. (2012). Radiation effects on MHD flow past an impulsively started vertical plate with variable heat and mass transfer. Int. J. of Appl. Math. and Mech., 8 (1), 66-85.

[33] Mukhopadhyay S., Bhattacharyya K., Layek G. C. (2011). Steady boundary layer flow and heat transfer over a porous moving plate in presence of thermal radiation. Int. J. Heat Mass Transfer, 54, 2751-2757.

[34] Cramer K. R. and Pai S. I. (1973). Magneto fluid dynamics for engineers and applied physicists McGraw Hill Book Company, New York. 